\title{
The BeppoSAX sub-second bursts project
}

\author{
M.J.S. Smith ${ }^{1,2}$, G. Gandolfi ${ }^{1}$, G. Celidonio ${ }^{1}$, A. Coletta $^{1}$, G. D’Andreta ${ }^{1}$, L. Di Ciolo ${ }^{1}$, A. Paolino ${ }^{1}$, R. Ricci ${ }^{1}$,

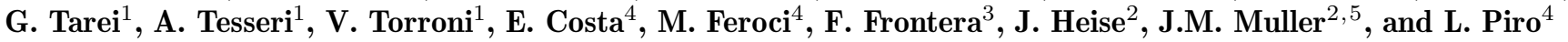 \\ 1 BeppoSAX Scientific Operation Centre, Telespazio s.p.a., Via Corcolle 19, 00131 Roma, Italy \\ 2 Space Research Organization Netherlands, Sorbonnelaan 2, 3584 TA Utrecht, The Netherlands \\ 3 Istituto di Tecnologie e Studio delle Radiazioni Extraterrestri (CNR), Via Gobetti 101, 40129 Bologna, Italy \\ 4 Istituto di Astrofisica Spaziale (CNR), Area Ricerca Roma Tor Vergata, Via del Fosso del Cavaliere, 00133 Roma, Italy \\ 5 BeppoSAX Science Data Centre, Telespazio s.p.a., Via Corcolle 19, 00131 Roma, Italy \\ e-mail: socbox@saxnet.sdc.asi.it
}

Received January 21; accepted June 22, 1999

\begin{abstract}
Sub-second GRBs have been identified as a separate class of gamma ray transients. However, despite recent advances in the field, sub-second GRB counterparts have yet to be found successfully. In order to explore the nature of these fast transients an on-line procedure is to be implemented at the BeppoSAX Scientific Operation Centre. The main objectives are the identification of true events and rapid position determination of their X-ray counterparts.
\end{abstract}

Key words: gamma-rays: bursts

\section{Introduction}

A key feature of gamma-ray burst (GRB) phenomena is their bimodal duration distribution, which separates GRBs into two classes of events: those with durations shorter and longer than $2 \mathrm{~s}$. The GRB durations are found to be anti-correlated with their spectral hardness ratios, the shorter GRBs being predominantly harder than the longer ones. Events in both classes, however, have the same peak intensity range and the same isotropic and inhomogeneous spatial distribution (Kouveliotou et al. 1993). The 4B BATSE catalogue shows that the number of events shorter than $1 \mathrm{~s}$ comprises $\sim 20 \%$ of all GRBs, although the numerical consistency of the sample varies slightly in different catalogues, depending on the instrumental trigger bin size. The bimodality suggests different geometries of emission sites or even different physical models for the central engine of the burst, but despite recent results from BeppoSAX and BATSE, the origin of the duration distribution is yet to be explained coherently.

A counterpart identification of a sub-second GRB would therefore be a crucial addition to the understanding of the underlying processes in GRB phenomena, but as yet no successful identification has been possible. As has been shown in the case of longer duration GRBs, and as may be realistically expected for the short GRBs, the rapid detection of an X-ray afterglow is, at least currently, a crucial step in allowing a swift search for optical and radio counterparts.

The BeppoSAX satellite is a natural candidate for the real-time monitoring of the high energy sky. The on-line scientific operations at the BeppoSAX Scientific Operation Centre (SOC) (Coletta et al. 1998) have the potential to be modified in order to perform a quick-look study of the short bursts category with as main scientific objective the detection and rapid position determination of sub-second GRBs.

We present an in-progress procedure for sub-second GRB detection to be implemented at the BeppoSAX SOC. This new program is complementary to the current proven procedure for GRB and afterglow detection and will extend the quick-look capabilities to the high-time resolution data domain. Extended and systematic analysis of GRB monitor (GRBM) triggers (Feroci et al. 1997) and Wide Field Camera (WFC) (Jager et al. 1997) light curves will be performed in order to select and study the short burst category.

\section{Quick-look sub-second GRB analysis}

The BeppoSAX SOC GRB procedure relies on the presence of a trigger, either from the GRBM or from BATSE, which contains the approximate time of a possible GRB. Given a trigger, the initial step in the procedure involves the analysis of the scientific housekeeping data of the Phoswich Detector System (PDS) lateral shields. These ratemeter data are inspected primarily in order to ascertain the validity of the trigger. However, the maximum $1 \mathrm{~s}$ resolution of this data sets a similar lower limit to the GRB duration which can be effectively discriminated from the many background events or noise fluctuations. In order to extend the systematic quick-look detection of GRBs to the sub-second class of events, an analysis must be made at a higher temporal resolution (see Fig. 1). Indeed, in the BeppoSAX SOC sub-second bursts procedure an analysis of the high time resolution GRBM packets is foreseen 

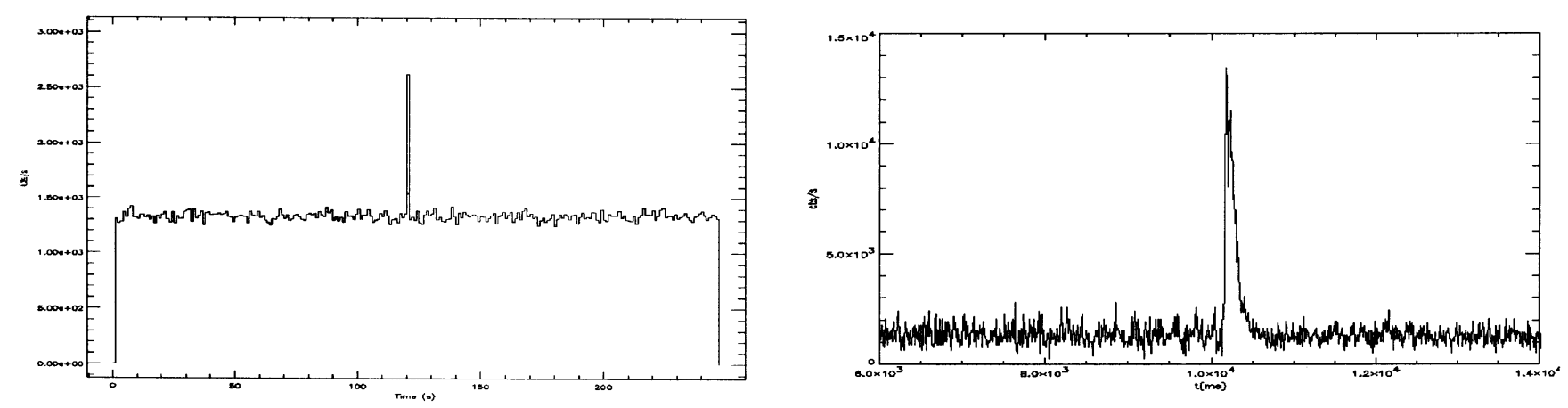

Fig. 1. Illustration of the necessity for the analysis of high-time resolution data in order to discriminate true bursts from background events. Both light curves are of the sub-second burst GRB 961122. On the left: PDS lateral shield ratemeter of $1 \mathrm{~s}$ resolution (4 minutes centered on trigger time). On the right: GRBM data of $8 \mathrm{~ms}$ resolution ( 8 seconds around trigger time)

with this aim in mind. The high time resolution packets contain lateral shield data with temporal resolution of up to $8 \mathrm{~ms}$, and are created on board whenever the GRBM trigger condition is fulfilled.

\section{Detection of sub-second GRB X-ray counterpart}

Any detection of GRB related X-ray emission is based on the analysis of WFC light curves. If positive, an image is made in order to determine the coordinates of the possible counterpart. At present, the WFC data analysis is performed up to a maximum temporal resolution of $1 \mathrm{~s}$. However, the study of a sample of longer GRBs with counterparts detected in the WFCs shows that this procedure might well fail to detect sub-second burst counterparts with similar peak fluxes (Feroci 1998). At quicklook level a minimum integrated flux is required to detect positively a source in a WFC celestial image, which thus poses a lower limit on the detectable fluence. In the case of a short burst, the X-ray flux will be concentrated in a sub-second time interval. Significant detection of the burst above background in the WFC light curve requires a bin size in the order of the burst duration, i.e. $\leq 1 \mathrm{~s}$. This demonstrates the need for an analysis of WFC light curves at higher temporal resolution in order to ensure an efficient detection of sub-second transients. To this end, the SOC software has been upgraded allowing analysis of WFC light curves at high time resolution and celestial images of sub-second integrations. This will form part of the new SOC procedure.

An analysis of sub-second WFC data in both a crowded and almost empty field has been performed. Preliminary results show that in either case the minimum required fluence for positive detection in a WFC image is $\sim 70$ counts (although the influence of background still requires further analysis). In the case of the near empty field the current SOC procedure would be adequate to detect a possible sub-second counterpart in a WFC light curve and image. The new procedure, based on high time resolution analysis, would nevertheless improve the detection efficiency. However, in the case of the crowded field, only the high time resolution analysis would allow the detection of counterparts in WFC images. The reason is that in this case the higher background (created by all the other sources in the field of view) requires a sub-second bin size for significant detection of the burst in the light curve.

An upper limit to the number of sub-second GRB counterparts which can be detected in WFC images may be obtained by converting the above mentioned minimum $\mathrm{X}$-ray fluence into the corresponding $\gamma$-ray fluence. Using a realistic GRB spectrum in the $2-25 \mathrm{keV}$ band consisting of a power law with photon index 1.35 (Feroci 1998), and applying the average ratio of X-ray $/ \gamma$-ray fluences of 0.24 (Strohmayer et al. 1998) to sub-second GRBs we find, as a rough estimate, a $\gamma$-ray fluence threshold of $4.010^{-8} \mathrm{ergs} / \mathrm{cm}^{2}(40-700 \mathrm{keV})$. Only more fluent GRBs may be detected in WFC images at BeppoSAX SOC. This includes $84 \%$ of sub-second bursts in the 4B BATSE catalogue.

\section{Conclusions}

A new quick-look procedure, based on high time resolution analysis of GRBM and WFC data, will be implemented at the BeppoSAX SOC in order to identify sub-second GRBs. We have shown that the detection of any sub-second X-ray counterpart in the WFC light curves and images will be maximized through the use of the upgraded software.

\section{References}

Coletta A., et al., 1998, Nuclear Physics B (Proc. Suppl.), 69/13,712

Feroci M., et al., 1997, Proc. SPIE 3114, 186

Feroci M., 1998 (private communication)

Jager R., et al., 1997, A\&AS 125, 557

Kouveliotou C., et al., 1993, ApJ 413, L101

Paciesas W.S., et al., 4th BATSE Burst Catalog available at http://cossc.gsfc.nasa.gov/cossc/batse

Strohmayer T.E., et al., 1998, ApJ 500, 873S 